\title{
A Review: Competence, Compromise, and Concomitance-Reaction of the Host Cell To Toxoplasma gondii Infection and Development
}

\author{
Author(s): Hong-Juan Peng, Xiao-Guang Chen , and David S. Lindsay \\ Source: Journal of Parasitology, 97(4):620-628. 2011. \\ Published By: American Society of Parasitologists \\ DOI: http://dx.doi.org/10.1645/GE-2712.1 \\ URL: http://www.bioone.org/doi/full/10.1645/GE-2712.1
}

BioOne (www.bioone.org) is a nonprofit, online aggregation of core research in the biological, ecological, and environmental sciences. BioOne provides a sustainable online platform for over 170 journals and books published by nonprofit societies, associations, museums, institutions, and presses.

Your use of this PDF, the BioOne Web site, and all posted and associated content indicates your acceptance of BioOne's Terms of Use, available at www.bioone.org/page/terms_of_use.

Usage of BioOne content is strictly limited to personal, educational, and non-commercial use. Commercial inquiries or rights and permissions requests should be directed to the individual publisher as copyright holder. 


\title{
A REVIEW: COMPETENCE, COMPROMISE, AND CONCOMITANCE-REACTION OF THE HOST CELL TO TOXOPLASMA GONDII INFECTION AND DEVELOPMENT
}

\author{
Hong-Juan Peng*, Xiao-Guang Chen*, and David S. Lindsay $†$ \\ Department of Etio-biology, School of Public Health and Tropical Medicine, Southern Medical University, 1838 North Guangzhou Rd, Guangzhou, \\ Guangdong Province 510515, People's Republic of China. e-mail: lindsayd@vt.edu
}

\begin{abstract}
Toxoplasma gondii is an important zoonotic parasite with a worldwide distribution. It infects about one-third of the world's population, causing serious illness in immunosuppressed individuals, fetuses, and infants. Toxoplasma gondii biology within the host cell includes several important phases: (1) active invasion and establishment of a nonfusogenic parasitophorous vacuole in the host cell, (2) extensive modification of the parasitophorous vacuolar membrane for nutrient acquisition, (3) intracellular proliferation by endodyogeny, (4) egress and invasion of new host cells, and (5) stage conversion from tachyzoite to bradyzoite and establishment of chronic infection. During these processes, $T$. gondii regulates the host cell by modulating morphological, physiological, immunological, genetic, and cellular biological aspects of the host cell. Overall, the infection/development predispositions of $T$. gondii-host cell interactions overtakes the infection resistance aspects. Upon invasion and development, host cells are modulated to keep a delicate balance between facilitating and eliminating the infection.
\end{abstract}

Toxoplasma gondii is an apicomplexan parasite capable of propagating asexually by endodyogeny in most nucleated cells. Approximately one-third of the world's human population is estimated to be chronically infected and have $T$. gondii tissue cysts containing bradyzoites, making it one of the most successful parasites infecting humans (Persson et al., 2007; Laliberté and Carruthers, 2008). Most postnatally acquired infections of $T$. gondii are not symptomatic in immunocompetent individuals. However, primary $T$. gondii infections may become widely disseminated, causing damage to the brain, eyes, liver, lungs, and lymph nodes and even causing death in immunecompromised individuals such as Acquired Immune Deficiency Syndrome patients and cancer patients undergoing immunosuppressive therapy. In pregnant women, $T$. gondii may be transmitted to the fetus transplacentally, resulting in tissue destruction and hence developmental defects in the fetus or newborn. Toxoplasma gondii is also an economically important parasite of livestock (Dubey et al., 1998).

Infection by $T$. gondii consists of an acute phase followed by a chronic phase. Infectious stages consist of sporozoites, tachyzoites, and bradyzoites. Felines, including domestic cats, act as definitive hosts of $T$. gondii within which the parasite completes the sexual phase of its lifecycle. Oocysts released in the feces undergo sporulation to form sporocysts that become a source of infection for intermediate hosts that include a wide range of domestic animals and humans. When oocysts are ingested by the intermediate host through contamination of water or food, sporocysts pass through the stomach and excyst in the intestine to release sporozoites. Sporozoites released from sporocysts in the intestine invade epithelial cells and differentiate into tachyzoites, an actively replicating form of $T$. gondii. Tachyzoites, due to their active multiplication, cause extensive tissue damage, may disseminate to different tissues of the host, and are also transplacentally (=horizontally) transmitted. Eventually, tachyzoites undergo stage conversion and become slowly multiplying bradyzoites within the tissue cyst. If for some reason the tissue

Received 3 December 2010; revised 29 January 2011; accepted 11 February 2011.

* These authors contributed equally to this paper.

$\dagger$ To whom correspondence should be addressed: Department of Biomedical Sciences and Pathobiology, Center for Molecular Medicine, Virginia-Maryland Regional College of Veterinary Medicine, 1410 Prices Fork Road, Virginia Tech, Blacksburg, Virginia 24061.

DOI: 10.1645/GE-2712.1 cyst becomes activated, then bradyzoites can undergo stage conversion and become tachyzoites (bradyzoites are the only stage that can infect feline enterocytes and lead to oocyst production, but that is beyond the scope of this review; Dubey et al., 1998). Acute infection consists of a cycle of host cell invasion by tachyzoites, replication of tachyzoites inside the parasitophorous vacuole (PV), and lytic egress from host cells. Chronic infection encompasses stage conversion of tachyzoites to bradyzoites and modification of the PV membrane (PVM) into a tissue cyst wall and maintenance of the tissue cyst with enclosed latent bradyzoites. During these processes, the host cells are competent to $T$. gondii attachment and invasion. Finally, the host cells then compromise with $T$. gondii, establishing a balance between facilitating and eliminating infection. Last, the host cells and the parasite are concomitant by the establishment of a chronic infection, resulting in tissue cysts. The present review highlights how host cells respond to the acute infection with $T$. gondii tachyzoites during invasion, intracellular proliferation, egress, and the establishment of chronic infection with bradyzoites in a competent/susceptible, compromised, and concomitant way.

\section{Toxoplasma gondii actively invades nonphagocytic and phagocytic cells}

The process of $T$. gondii invasion into host cells is different from phagocytosis as it involves active penetration assisted by the actomyosin motor system of the parasite (Dobrowolski and Sibley, 1996; Black and Boothroyd, 2000) (Figs. 1 and 2). Differences occur between nonphagocytic and phagocytic cells, but the mechanisms are the same except for some modifications, which occur on the cell membrane of phagocytes. Nevertheless, $T$. gondii can be actively phagocytosed by competent phagocytic cells (Pacheco-Soares and De Souza, 2000; Li et al., 2008; Walker et al., 2008).

Using cultured cells, neutrophils, even those not in contact with parasite, are activated to form membrane ruffles, filopodia (these filopodia migrate over the parasite surface), or tunnel-like invaginations on the membrane near the site of penetration; these reactions are helpful for $T$. gondii invasion (MacLaren et al., 2004). There are several kinds of interactions between $T$. gondii tachyzoites and neutrophils before internalization. First, many fingerlike filopodial projections of the host cell make contact with the lateroposterior portion of the parasite, and roughly $59 \%$ of tachyzoites interact with neutrophils in this manner. Second, about $25 \%$ of tachyzoites attach to the surface of neutrophils with 




Figure 1. Diagram of a host cell being hijacked to be competent, compromised, and concomitant to Toxoplasma gondii. (I) Toxoplasma gondii actively invades nonphagocytic and phagocytic cells. (II) The parasitophorous vacuole (PV) protects T. gondii from being eliminated by host cells, especially from endosomal acidification and lysosomal fusion. (III) The host cell is induced to be competent to T. gondii infection and allow its proliferation by controlling the host cell cycle. The host cell is induced to arrest at S-phase for T. gondii invasion and induced into entering G2/M phase for $T$. gondii proliferation. (IV) The host cell provides a nutrient pool for $T$. gondii proliferation and replication. Toxoplasma gondii elaborates pores in the PV membrane (PVM) to change its permeability, allowing small molecules to diffuse across the PVM. The other nutrients such as cholesterol are translocated along host microtubules (MTs) to the PV, and invaginated intact into PVM through HOST structures containing host MTs. After nutrients enter the PV, they likely enter into the parasite by membrane transporters. (V) The host cell is exploited by $T$. gondii to establish an invasion, replication, and egress competent chemical (Ion) environment for the parasite. (VI) The host cell cytoskeleton is reorganized to facilitate PV formation and T. gondii invasion. Microtubules form circular, basket-like structures that surround the PV in infected cells. The intimate interactions between $T$. gondii and host microtubules result in suppression of cell division and/or cause a mitotic defect, thus providing a larger space for parasite multiplication. (VII) Host cell anti-apoptotic reactions are induced by $T$. gondii for both self-protection and parasite-protection, mainly through regulating the death receptor pathway, the NF-kB pathway, and the PI3K pathway. (VIII) Toxoplasma gondii infection induces several successful immune evasion mechanisms by the host cell. Toxoplasma gondii induces blockage of IFN- $\gamma$ signaling pathway to control chronic and latent infection or induces the infected host cells to be less responsive to IFN- $\gamma$-induced upregulation of many genes, including MHC Class II, iNOS, and the p47 GTPases. (IX) Concomitance of host cell and T. gondii appears with the conversion of tachyzoites into bradyzoites in immunocompetent hosts. Abbreviations: T: tachyzoite; B: bradyzoite; HN: host nucleus; PVM: parasitophorous vacuole membrane; MT: microtubule; MF: microfilament; mt: mitochondria; SM: small molecule; H.O.S.T: host organelle-sequestering tubulo structures.

a protruded conoid, through the filopodia, or directly through the conoid-host cell interface, where secretion seems to be occurring. The tachyzoite enters a neutrophil by the anterior (conoidal) end, which embraces it with filopodia or by forming a glovelike tunnel around the anterior portion of the tachyzoite, and by the odd formation of a cavity in a lymphocyte. Third, approximately $15 \%$ of tachyzoites are internalized by the neutrophils with the posterior end first (MacLaren et al., 2004).

Toxoplasma gondii contains 3 special sets of secretory organelles that help the parasite to gain entry into host cells. These include cigar-shaped micronemes (MICs), bulb-shaped rhoptries, and spherical dense granules. Micronemes and rhop- tries are located near the apical end, and dense granules are scattered throughout the parasite. The contents of these organelles are released at specific time points during invasion, and hence host cell entry by $T$. gondii is a well-orchestrated event. The process of host cell invasion by $T$. gondii begins with transient attachment of the parasite to the host cell. Then, a calciummediated signaling event results in secretion of MIC to the parasite surface that enables the parasite to establish a firm interaction with the host cell. However, many of the host cell receptors involved in parasite entry into host cell remain undefined (Saffer et al., 1992; Dubremetz and Schwartzman, 1993; Boothroyd and Dubremetz, 2008; Breinich et al., 2009). 


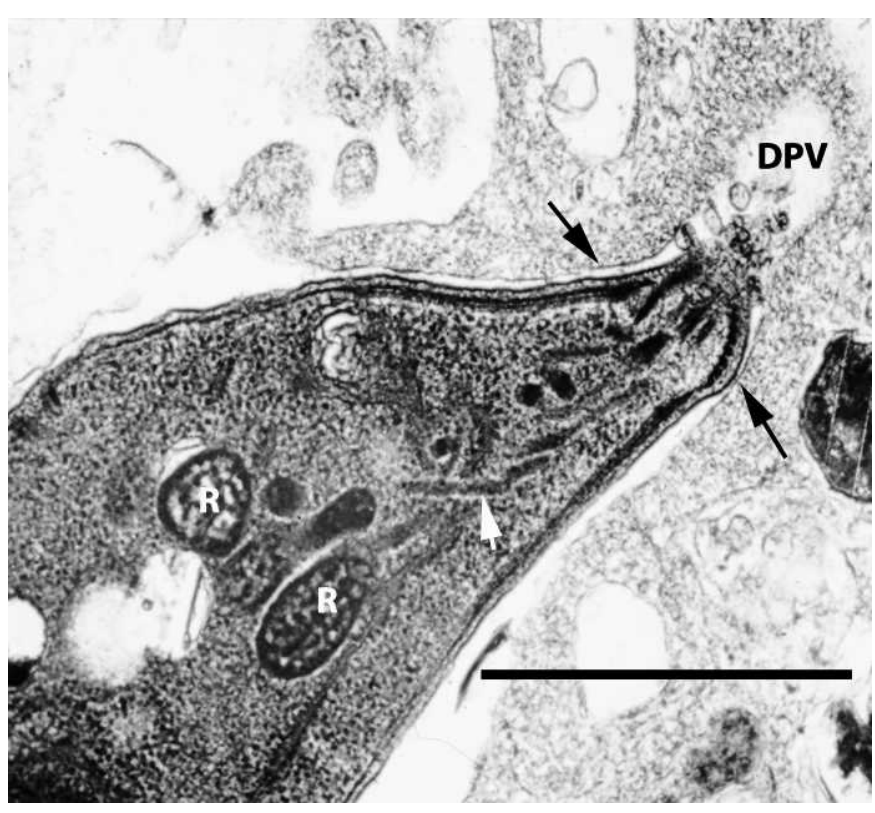

Figure 2. Tachyzoite of the RH strain of $T$. gondii penetrating into a human fibroblast cell and establishing a developing parasitophorous vacuole (DPV). Note the association of the host cell membrane (black arrows) with the conoidal end of the tachyzoite. Rhoptry sacks $(\mathbf{R})$ and a rhoptry neck (white arrow) are visible. Bar $=1.0 \mu \mathrm{m}$.

\section{The parasitophorous vacuole (PV) protects $T$. gondii from being eliminated by the host cell}

Toxoplasma gondii dwells inside a PV once it gains entry into a host cell (Figs. 3 and 4). The formation of the PV is closely linked to the establishment of a moving junction (MJ) between the host cell and tachyzoites during invasion. The MJ forms when $T$. gondii attaches to receptors on the host cell and secretes MIC and rhoptry neck proteins (RONs), which assemble at the interface of the parasite and the host cell surface (Alexander et al., 2005; Cao et al., 2009). Recent studies have identified some key components of MJ that include microneme protein AMA1 and RON proteins RON 2, RON4, RON5, and RON8 (Besteiro et al., 2009). After establishing the moving junction, the parasite pushes itself forward, and the invagination of the plasma membrane around the invading parasite results in the formation of the PV (Fig. 2). Through the MJ, the parasite actively excludes incorporation of many of the host transmembrane proteins into the PV membrane, while allowing GPI anchored proteins to remain. Toward the end of the invasion process, the pinching off of the PVM results in separation from the host cell plasma membrane; at this point, the intracellular parasite is enclosed within the PVM within which it spends its intracellular life (Figs. 3-5). After invasion, the parasite further modifies the PVM, by inserting novel proteins derived from its secretory organelles, the rhoptries and the dense granules (Joiner and Roos, 2002; Straub et al., 2009). During penetration, the parasite injects many rhoptry proteins into the host cell cytosol that appear like small satellite vesicles and are referred to as evacuoles that eventually fuse with the PVM (Hakansson et al., 2001). As the invading parasite effectively excludes many of the transmembrane proteins of the host cell that are involved in fusion with lysosomes and most of the PVM is derived from the

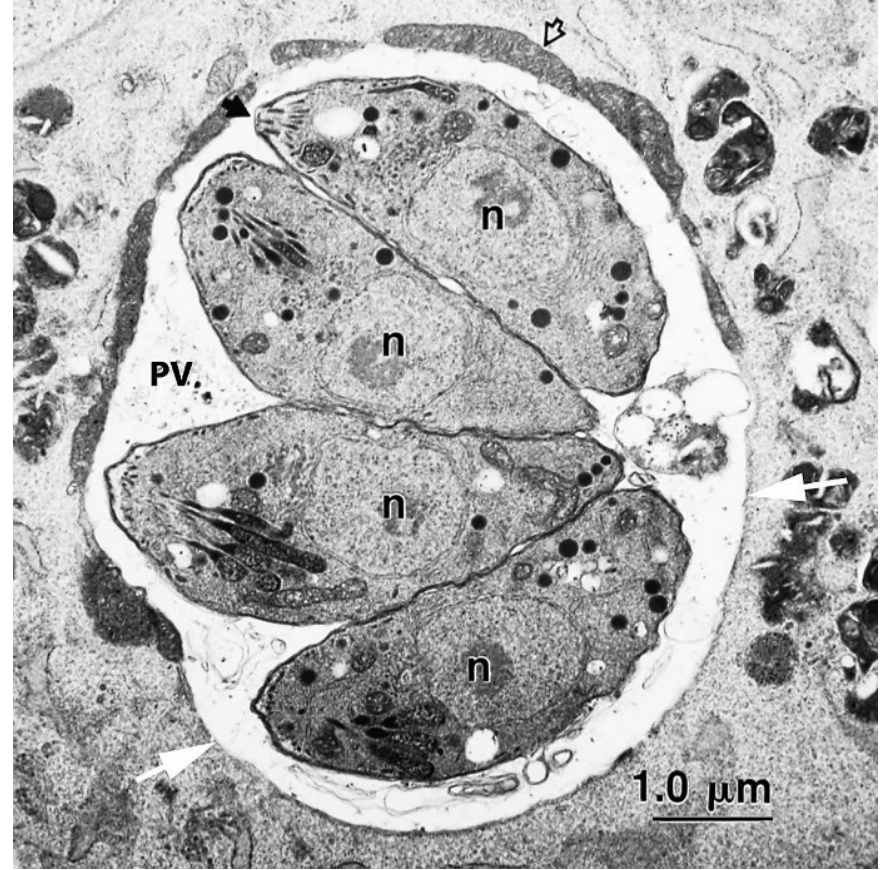

FIgURE 3. Group of $4 \mathrm{~T}$. gondii tachyzoites developing in a parasitophorous vacuole (PV) surrounded by a parasitophorous vacuole membrane (white arrows). Host cell mitochondria (open arrow) are associated with the parasitophorous vacuole membrane. The nucleus (n) of the tachyzoites is labeled, and the conoid (arrow) is visible in 1 tachyzoite. Bar $=1.0 \mu \mathrm{m}$.

host cell, the PVM is a nonfusogenic compartment that is resistant to acidification by the endosome-lysosomal system of the host cell (Mordue, Desai, and Dustin, 1999; Mordue, Hakansson, et al., 1999; Charron and Sibley, 2004). After entry into the host cell, the PVM closely associates with host mitochondria (Figs. 3, 4) and endoplasmic reticulum (ER) and migrates toward the nucleus using the host microtubule network (Sibley et al., 1994).

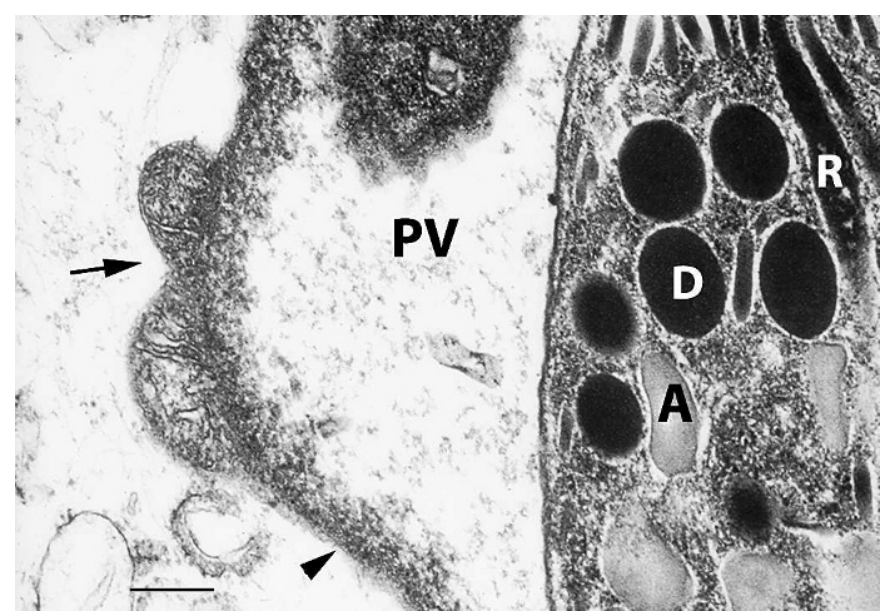

FIGURE 4. Mitochondrion (arrow) in close association with a developing tissue cyst of $T$. gondii. A bradyzoite in the parasitophorous vacuole (PV) showing rhoptries (R), dense bodies (D), and amylopectin granules $(\mathbf{A})$. Bar $=0.25 \mu \mathrm{m}$. 


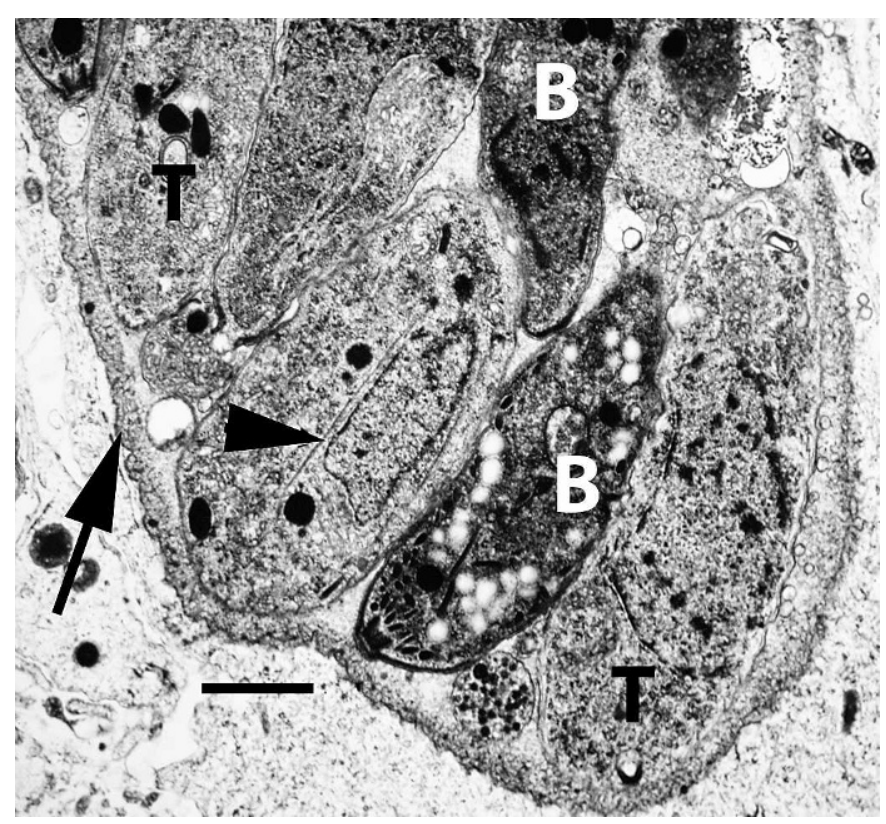

FIGURE 5. Young tissue cyst of the RH strain of T. gondii induced with decoquinate demonstrating bradyzoites (B) and tachyzoites (T). The arrowhead points to a daughter tachyzoite developing by endodyogeny within a tachyzoite. Note the tissue cyst wall (arrow) surrounding the organisms. Bar $=1.0 \mu \mathrm{m}$.

\section{Toxoplasma gondii manipulates the cell cycle of the host cell for effective infection and intracellular proliferation}

Toxoplasma gondii can hijack host cells by modulating their cell cycle. Thus, $T$. gondii infection induces human fibroblast monolayers to transit from the G0/G1 to S-phase and remain arrested in the S-phase of the cell cycle (Molestina et al., 2008). This transition allows efficient invasion by the parasite, as $T$. gondii has been shown to attach and invade S-phase host cells more readily than cells in other phases of the cell cycle (Lavine and Arrizabalaga, 2009). Interestingly, growth media from $T$. gondii infected cells have also been shown to induce noninfected cells to enter S-phase. This suggests that $T$. gondii directly releases or stimulates infected host cells to release a soluble factor into the culture medium that induces host cells to enter S-phase and thus facilitate efficient infection (Lavine and Arrizabalaga, 2009). There is a preferential attachment of $T$. gondii to S-phase cells compared to G1-phase, and it is not dependent on the cells being in synchronous cycles at the time of infection (Grimwood et al., 1996).

Once inside the host cell, tachyzoites induce the host cell to arrest at the $\mathrm{G} 2 / \mathrm{M}$ phase of the cell cycle to enable parasite proliferation. Toxoplasma gondii can divide only in host cells arrested in G2 phase in which mitosis has been prevented (Brunet et al., 2008; Molestina et al., 2008). A cell cycle regulating host gene UHRF1 is required for intracellular $T$. gondii proliferation and induces host cell transition from $\mathrm{G} 1 / \mathrm{S}$ phase to $\mathrm{G} 2$ phase. RNAi-mediated silencing of UHRF1 causes the cell cycle to arrest in G1 phase, resulting in a significant reduction in parasite proliferation (Brunet et al., 2008).

\section{The host cell provides a nutrient pool for T. gondii during proliferation}

Transcription profiles and genome sequencing have revealed that $T$. gondii is auxotrophic for some metabolites (Blader et al.,
2001; Gail et al., 2001; Crawford et al., 2006). Being an obligate intracellular pathogen, $T$. gondii must acquire nutrients inside the host cell, which cannot be obtained from extracellular sources. The PVM not only surrounds the intracellular $T$. gondii and provides a stable environment for parasite proliferation but also is an exchange interface between host cell cytosol and the parasite. Toxoplasma gondii modifies PVM permeability by making pores in the PVM, allowing some small molecules that cannot be synthesized de novo by the parasite, such as glucose, arginine, iron, tryptophan, phospholipids, purine nucleosides, and cofactors, to diffuse across the PVM. Then these molecules enter the parasite by membrane transporters (Schwab et al., 1994, Charron and Sibley, 2002; Sehgal et al., 2005; Blader and Saeij, 2009).

In contrast to these small molecules that passively diffuse across the PVM, other nutrients are obtained by more active mechanisms. Toxoplasma gondii is deficient in the ability to synthesize sterol; blockade of exogenous and endogenous sources of host cholesterol reduces parasite replication. Intracellular proliferation of parasites is dependent on cholesterol captured from host cell lysosomes, through low-density lipoprotein (LDL)-mediated PV endocytosis, which is independent of vesicular fusion and requires parasite viability. The host microtubules (MTs) are reorganized upon $T$. gondii invasion, and the parasite is wrapped in a network of host MTs. The endo-lysosomes containing LDL-loaded cholesterol are translocated along host MTs to the PV and are placed into the PVM through host MTs containing a specific structure: host organelle-sequestering tubule structures (HOSTs). At this point, elongated membranous tubules containing LDLloaded cholesterol are formed. These membrane tubules are stabilized by the formation of a protein coat of parasite origin, including the dense granule protein GRA7 (Coppens et al., 2000, 2006).

The exact mechanism for entry of nutrients into the vacuole is unclear. The PV containing T. gondii is surrounded by an extensive tubular network system accumulated within the PV and in contact with the PVM. It has been speculated that the tubulovesicular network in the vacuolar space delivers these nutrients from the PVM or PV to the parasite, or reversely, from parasite to PV or PVM (Sibley et al., 1995; Lingelbach and Joiner, 1998).

Toxoplasma gondii can also recruit host mitochondria and ER in synthesizing and transporting some nutrients the parasite needs; this recruitment happens quickly after the parasite invades a cell (Sinai et al., 1997; Crawford et al., 2006). Even though lipoate can be synthesized de novo and used in the $T$. gondii apicoplast, the parasite can also scavenge lipoate from the host cell, and the host-derived lipoate can be metabolized in the parasite's mitochondria (Crawford et al., 2006). This phenomenon may indicate a deficiency in lipoate metabolism in $T$. gondii, which may be due to the lack of a lipoate transporter required to transfer the nutrient out of the apicoplast (Crawford et al., 2006).

The PVM has a close association with, or is tightly enshrouded by, host cell mitochondrial membranes and ER, and even endolysosomes (Lindsay, Mitschler et al., 1993). It is speculated that host cell mitochondria and ER provide lipids and products of intermediary metabolism to the intracellular parasite (Sinai et al., 1997; Sinai and Joiner, 2001; Coppens et al., 2006). The tight association of the PVM with the mitochondria and ER also provides a key source of new phospholipids incorporated into the PVM, which plays an important role in the PVM enlargement (Sinai et al., 1997; Sinai and Joiner, 2001). 


\section{The host cell is exploited by $T$. gondii to establish an invasion-, replication-, and egress-competent chemical- ion environment}

Cellular chemical changes induce the host cell to become competent to invasion and egress by $T$. gondii. Mobilization of intracellular $\mathrm{Ca}^{2+}$ plays an important role in facilitating parasite invasion and egress events. When tachyzoites are attaching to host cells, an undefined signaling event results in mobilization of $\mathrm{Ca}^{2+}$ from the parasite ER that triggers the invasion process (Pingret et al., 1996; Lovett and Sibley, 2003; Li et al., 2008). An elevation of $\left[\mathrm{Ca}^{2+}\right] \mathrm{i}$ is also associated with rapid egress of parasites from the host cells at the end of intracellular proliferation. Calcium ionophores have been used to induce the release of the parasites from infected cells even in the early periods $(2 \mathrm{hr})$ postinvasion (Caldas et al., 2007; Kafsack et al., 2009)

Toxoplasma gondii invasion causes elevations of $\left[\mathrm{Ca}^{2+}\right] \mathrm{i}$ and arachidonic acid (AA), which are from both host cells and parasites. $\left[\mathrm{Ca}^{2+}\right] \mathrm{i}$ is an essential factor for protozoan parasite differentiation, cytoskeleton dynamics, motility, and cell growth (Li et al., 2008). During $T$. gondii invasion, the rise of $\left[\mathrm{Ca}^{2+}\right] \mathrm{i}$ concentration in host cells depends on the activation of phospholipase C (PLC) on the host cell membrane, consequently triggering the PLC-PKC signaling pathway, which results in the flowing of extracellular $\mathrm{Ca}^{2+}$ and the releasing of intracellular $\mathrm{Ca}^{2+}$ pools. Elevated $\left[\mathrm{Ca}^{2+}\right] \mathrm{i}$ induces agglutination of host cell microfilaments ( $\mathrm{Li}$ et al., 2008). Phospholipase $\mathrm{C}$ hydrolyzes phosphatidylinositol bisphosphate (PIP2), producing inositol triphosphate and 2-diacylglycerol. Inositol triphosphate is a secondary messenger, which also can regulate the releasing of the $\mathrm{Ca}^{2+}$ in cells (Lovett et al., 2002). Two-diacylglycerol will induce the release of $\mathrm{Ca}^{2+}$ attached to the ER membrane (Rodriguez et al., 1995; Salter and Hicks, 1995). Arachidonic acid is a second messenger, which is produced by phospholipase A and mediates decomposition of phospholipids on the cell membrane (Ronco et al., 2002). Arachidonic acid mediates multiple cellular activities such as cell apoptosis and inflammation. The concentration of AA is 8.44-fold and 5.02-fold of the basal concentration in the supernatant of phagocytic (J774A.1) and nonphagocytic cells (L929), respectively, after T. gondii infection ( $\mathrm{Li}$ et al., 2008). Toxoplasma gondii can produce secretory and cytosolic Phospholipase A2 (PLA2), and the production is closely related to $T$. gondii virulence (Buitrago-Rey et al., 2002). PLA2 inhibitors can significantly decrease the intracellular AA concentration and the infectivity of $T$. gondii tachyzoites.

The host cell ER fuses with the PVM prior to PV disruption. Fusion of ER with the PVM triggers the release of calcium into the PV, which may also be the mechanism underlying intravacuolar parasite movement and IFN- $\gamma$-induced parasite egress (Melzer et al., 2008).

Two recently published papers demonstrate the role of parasiteencoded perforin-like protein 1 and host-derived calpain in parasite escape from the host cell (Chandramohandas et al., 2009; Kafsack et al., 2009). Both of these egress mechanisms are regulated by calcium. Perforin-like protein 1 localizes to $T$. gondii micronemes and is secreted in a calcium-dependent manner. Perforin-like protein 1-deficient $T$. gondii loses the ability to rapidly permeabilize the PVM and host cell membrane during egress. This results in a failure to exit normally after proliferation in PV, resulting in entrapment of tachyzoites within host cells (Kafsack et al., 2009). However, it also has been shown that in addition to parasite proteins, host-derived factor calpain plays an essential role in $T$. gondii egress. A calcium signal triggered late during $T$. gondii infection activates host cell calpain, which re-localizes to the host cell plasma membrane, cleaving cytoskeletal proteins to facilitate parasite egress (Chandramohanadas et al., 2009)

\section{The host cell cytoskeleton is reorganized fitting to $T$. gondii invasion and PV formation}

Invasion of $T$. gondii can activate the reorganization of cytoskeleton elements such as microfilaments and microtubles of the host cell (da Silva et al., 2009). Interference of host cell actin dynamics with cytochalasins and jasplakinolide partially blocks T. gondii entrance into cells (Ferreira et al., 2003). Microfilament adherence and accumulation has also been observed during $T$. gondii invasion of phagocytic cells, but not non-phagocytic cells (Li et al., 2008). Apparently, microfilaments function differently in phagocytic and non-phagocytic cells during $T$. gondii invasion. The agglutination of microfilaments in phagocytic cells plays an important role for the successful invasion by $T$. gondii (Li et al., 2008). Some studies have suggested that host MTs may not be involved in T. gondii invasion (Dobrowolski and Sibley, 1996; Li et al., 2008). It has been reported that host cell MTs are involved in the development and enlargement of the PV (Andrade et al., 2001). A recent report has shown that there is indeed reorganization of MTs during parasite entry (Sweeney et al., 2010). Treatment of host cells with colchicine (a MT inhibitor) changes the shape of the PV (Melo et al., 2001). Recruitment of T. gondii is greatly reduced in cells treated with colchicum and cytochalasin $\mathrm{D}$, including phagocytic and non-phagocytic cells. This result may due to the inhibition of MT movement, resulting in the impairment of resistance of phagocytic lysosome fusion of the PVM enclosing intracellular T. gondii (Andrade et al., 2001; Li et al., 2008).

Remodeling of the host cell MT network upon $T$. gondii invasion in several different host cells has been reported. The phenomenon of remodeling is not cell type specific. Microtubules in uninfected host cells radiate from the host nucleus and form an ordered network throughout the cytoplasm. Nevertheless, in infected cells, microtubules form a circular basket-like structure that surrounds the PV. The remodeling of MTs is dependent on parasite viability and infection time. The intimate interactions between $T$. gondii and host MTs results in suppression of host cell division or causes a mitotic defect, or both, thus providing a larger space for parasite multiplication (Walker et al., 2008).

The small GTPase of ARF6 from the host cell is recruited to the site of entry and plays an important role in $T$. gondii invasion through the activation of PI3-kinase, with the involvement of PIP2 and PIP3. RNAi-mediated ARF6 gene knockdown greatly reduces the recruitment of tachyzoites in Vero cells. This indicates that the host cell cytoskeleton is involved in parasite invasion and plays an important role, as ARF6 regulates membrane trafficking and actin cytoskeleton reorganization (da Silva et al., 2009). Maintenance of the integrity of the host cell actin cytoskeleton is important in parasite invasion (da Silva et al., 2009).

\section{The host cell anti-apoptotic reactions are induced by T. gondii for both self-protection and parasite protection}

Toxoplasma gondii can infect all nucleated cells and induces little obvious disturbance during parasite multiplication prior to host cell rupture. The ability to extend the life of infected host 
cells is an anti-apoptosis-induced reaction triggered by $T$. gondii infection. Interference of $T$. gondii with host cell apoptosis is related to virulence factors of $T$. gondii strains (Angeloni et al., 2009) and depends on the cell types being infected (Vutova et al., 2007; Hippe et al., 2008). The mechanisms are mainly through regulating the death receptor (Hippe et al., 2008) and the NF-кB (Payne et al., 2003) and the PI3K pathways (Yang et al., 2004).

Toxoplasma gondii inhibits death receptor-mediated apoptosis in host cells (Goebel et al., 2001; Payne et al., 2003; Vutova et al., 2007). Fas/CD95 triggers an apoptotic cascade that is crucial for immunity and the outcome of infectious diseases. However, $T$. gondii counteracts this death receptor-mediated cell death-Fas/ CD95 cascade differently in type I and type II host cells. In type I host cells (like SKW6.4 cells), T. gondii blocks this apoptotic cascade directly through interference with caspase 8 . In type II host cells (like HeLa cells), T. gondii significantly reduces Fas/ CD95-triggered apoptosis by inhibiting the activities of initiator caspases 8 and 9 and effector caspase 3/7, by decreasing the apoptogenic function of mitochondria at the mitochondrial amplification loop (Vutova et al., 2007; Hippe et al., 2008).

The anti-apoptotic reaction induced by $T$. gondii infection is also associated with upregulation of a series of anti-apoptotic genes, such as the activation of NFKB-dependent anti-apoptotic genes (Molestina et al., 2003; Payne et al., 2003). PKB inactivates the Forkhead family transcription factor FKHR1, which regulates apoptosis-inducing genes, reduces activation of caspase molecules, and downregulates poly ADP-ribose polymerase expression (Goebel et al., 2001). PKB has also been implicated in positive regulation of the $\mathrm{NF \kappa B}$ signaling pathway that leads to induction of several survival-promoting genes (Patra et al., 2004). The anti-apoptotic function of PKB occurs in part through phosphorylative inactivation of the proapoptotic proteins $\mathrm{Bad}$ and caspase-9 (Blume-Jensen et al., 1998).

The phosphoinositide 3-kinase (PI 3-kinase) pathway and immediate downstream effector protein kinase B (PKB/Akt) play important roles in cell survival and apoptosis inhibition (Scheid and Woodget, 2001; Yang et al., 2004). Toxoplasma gondii activates PI3-kinase through the signal pathway transmitted by the heterotrimer of Gi protein, resulting in the phosphorylation of PKB/Akt and ERK1/2 MAPK. Both in vitro and in vivo experiments have demonstrated that after mouse phagocytes are infected by $T$. gondii, the PKB/Akt pathway is activated, and the induction of apoptosis in infected macrophages is prevented (Kim and Denkers, 2006).

Toxoplasma gondii appears to block apoptosis in its host cells at different points and to co-opt host apoptotic signaling pathways in an environment-sensing mechanism (Persson et al., 2007). The cells infected by $T$. gondii are resistant to multiple inducers of apoptosis, including cytotoxic $\mathrm{T}$ lymphocyte (Fas-dependent or -independent), IL-2 deprivation, gamma irradiation, UV irradiation, and the calcium ionophore beauvericin (Nash et al., 1998; Luder and Gross, 2005). However, T. gondii blocks host cell apoptosis, including the inhibition of cytochrome $\mathrm{C}$ released from host cell mitochondria, upregulation of anti-apoptotic proteins of the Bcl-2 and IAP families, interference with proapoptotic Bax and $\mathrm{Bad}$, modulation of cell death-regulating kinase activities, inhibition of caspase, and possibly modulation of cytochrome Cinduced caspase 3/7 activation (Hippe et al., 2008).

Usually $T$. gondii triggers host cell rupture and egress of parasites before induction of host cell apoptosis (Persson et al.,
2007). Death receptor-induced egress of $T$. gondii depends on caspase-mediated release of intracellular calcium early in the apoptotic cascade. $\mathrm{T}$ cells induce rapid egress of infectious parasites by acting on infected cells via death receptor- or perforin-dependent pathways (Persson et al., 2007).

\section{Toxoplasma gondii infection induces a series of successful immune evasion mechanisms in the host cell}

Successful parasitic infection with host and parasite survival necessitates a balance between parasite immune evasion and host immune surveillance. Upon invasion, T. gondii induces a strong cellmediated and IFN- $\gamma$-driven immune response in its mammalian hosts to resist acute infection and maintain latent infection (Suzuki et al., 1989). However, T. gondii also adjusts the host immune response by inducing infected cells to be less responsive to IFN- $\gamma$ signaling. This ability may be the most important mechanism for immune evasion from a robust IFN- $\gamma$ mediated cellular immunity employed by $T$. gondii (Kim et al., 2007). For example, host cells infected by $T$. gondii are significantly less responsive to IFN- $\gamma-$ induced upregulation of many genes, including MHC Class II, iNOS, and the p47 GTPases. IFN- $\gamma$-inducible gene expression of iNOS, MIG, IIGP1, and IFN- $\gamma$-induced NO is inhibited in the infected cells (Lang et al., 2006; Zimmermann et al., 2006; Kim et al., 2007). The ability and inability of host cells to inhibit IFN- $\gamma$ responses is correlated with differences in host range, $T$. gondii virulence, and persistence (Kim et al., 2007). Toxoplasma gondii infection-induced IFN- $\gamma$ signaling is inhibited by blocking STAT1 transcriptional activity (Kim et al., 2007) or via a decrease in STAT1 tyrosine phosphorylation, or both (Zimmermann et al., 2006). There is some evidence that $T$. gondii inhibits STAT1 by upregulating levels of the endogenous suppressor of cytokine signaling protein (SOCS) in the host cells, which contributes to the parasite's inhibition of IFN- $\gamma$. SOCS represents a protein family, including SOCS1-7 and CIS, which are considered as attenuators of IFN- $\gamma$ signaling through either inhibiting the catalytic activity of JAKs or by inhibiting recruitment of STATs (Zimmermann et al., 2006). These findings show the inhibition of IFN- $\gamma$ signaling will result in immune evasion of $T$. gondii, but the mechanisms of these kinds of inhibitions are still not clear.

\section{Concomitant status between the host cell and $T$. gondii appears with the conversion of tachyzoites into bradyzoites in immunocompetent hosts}

After initial acquisition of infection in immunocompetent hosts, T. gondii tachyzoites soon convert into bradyzoites to establish a chronic, asymptomatic infection (Dubey et al., 1998). Bradyzoites normally cannot be cleared by the host because of the weak immune response that they elicit; elimination is also difficult because of their refractoriness to existing drugs (Guimarães et al., 2008). Thus, a concomitant status between the host and the parasite is established.

The mechanism for conversion from the acute to the chronic phase of infection is still largely unknown. Most isolates of $T$. gondii will produce tissue cysts in vitro (Lindsay, ToivioKinnucan, and Blagburn, 1993) despite the infective stage inoculated (Lindsay et al., 1991). A variety of environmental stresses will induce this conversion. Stress results in phosphorylation of $T$. gondii eukaryotic initiation factor- $2 \alpha(\operatorname{TgIF} 2 \alpha)$, and TgIF $2 \alpha$ phosphorylation is employed by cells to maintain a latent 
state. Phosphorylated TgIF $2 \alpha$ in bradyzoites might be part of the explanation for the manner in which these parasites maintain their quiescent state in the host cells (Narasimhan et al., 2008).

The tachyzoite-bradyzoite conversion models in vitro can be achieved using various stress conditions that mimic immunederived stressors, like high $\mathrm{pH}$, IFN- $\gamma, \mathrm{TNF}-\alpha$, nitric oxide, high temperature, nutrient starvation, or by some drugs used to treat T. gondii infections (Bohne et al., 1993; Soete et al., 1994; Lindsay et al., 1998; Fox et al., 2004). Stress-induced elevation of cyclic nucleotides may play an important role in the conversion of $T$. gondii tachyzoites to bradyzoites (Kirkman et al., 2001).

At the beginning of conversion, the expression of tachyzoitespecific genes such as SAG1, SAG2A, SAG2B, LDH1, ENO2, PtdIns(t), and SRS1-SRS3 are turned off and replaced by the expression of bradyzoite-specific genes such as SAG2C, SAG2D, SAG4, BSR4, MAG1, LDH2, ENO1, BAG1, PtdIns(b), and pATPase (Lyons et al., 2002). During stage conversion, there is a decrease in the expression of immunogenic surface proteins, and metabolic enzymes and an increase in the abundance of genes that act to facilitate entry into host cells (Lyons et al., 2002). Changes in host cell transcription can directly influence the molecular environment to enable bradyzoite development. Human cell division autoantigen-1 upregulation causes the inhibition of parasite replication and subsequently leads to tachyzoite-bradyzoite conversion. How the parasite modulates the biochemical environment of the cell is unknown, but, apparently, bradyzoites are primarily distributed in differentiated, long-lived cells such as mature skeletal muscle and brain neurons (Radke et al., 2003).

Interferon- $\gamma$ also drives the conversion of $T$. gondii tachyzoites to bradyzoites, resulting in the conversion of acute infection to chronic infection, and, at the same time, suppresses the reactivation of bradyzoites to tachyzoites in immune-competent hosts (Bohne et al., 1993).

The intracellular $T$. gondii extensively modulates its host cell so as to efficiently grow and divide. In doing so, it is one of the most successful parasites of humans, infecting almost one-third of the world's population. In immunocompetent hosts, $T$. gondii induces the host cell to be competent, compromised, and concomitant to the parasite to help in every way for $T$. gondii invasion, replication, and persistence. It remains mostly a mystery how the host cell is induced to be competent for $T$. gondii invasion and egress, compromised to $T$. gondii in parasitizing, proliferation, and multiplication, and, eventually, concomitant in the relationship between the parasite and host cell when tachyzoites successfully convert to bradyzoites.

\section{ACKNOWLEDGMENTS}

We thank Drs. R. Y. Gaji, J. Strobl, and Q. Han for comments on this manuscript. This work was supported by grants from the National Scientific Foundation of China (Key program: 31030066), National Special Research Programs for Non-Profit Trades (Agriculture) (200803017), Guangdong Province Universities and Colleges Pearl River Scholar Funded Scheme (2009) to X.-G.C., National Scientific Foundation of China (81071377), Guangdong Provincial Natural Science Foundation (10151051501000033), Research Fund for the Doctoral Program of Higher Education of China (20104433120014), and Guangdong Provincial Medical Research Foundation (A2010356) to H.-J.P.

\section{LITERATURE CITED}

Alexander, D. L, J. Mital, G. E. Ward, P. Bradley, and J. C. Boothroyd. 2005. Identification of the moving junction complex of
Toxoplasma gondii: A collaboration between distinct secretary organelles. PLoS Pathogens 1: e17.

Andrade, E. F., A. C. Stumbo, L. H. Monteiro-Leal, L. Carvalho, and H. S. Barbosa. 2001. Do microtubules around the Toxoplasma gondii-containing parasitophorous vacuole in skeletal muscle cells form a barrier for the phagolysosomal fusion? Journal of Submicroscopic Cytology and Pathology 33: 337-341.

Angeloni, M. B., N. M. Silva, A. S. Castro, A. O. Gomes, D. A. Silva, J. R. Mineo, and E. A. Ferro. 2009. Apoptosis and S phase of the cell cycle in BeWo trophoblastic and HeLa cells are differentially modulated by Toxoplasma gondii strain types. Placenta 30: 785-791.

Besteiro, S., A. Michelin, J. Poncet, J. F. Dubremetz, and M. Lebrun. 2009. Export of a Toxoplasma gondii rhoptry neck protein complex at the host cell membrane to form the moving junction during invasion. PLoS Pathog. 5: e1000309

Black, M. W., and J. C. Boothroyd. 2000. Lytic cycle of Toxoplasma gondii. Microbiology and Molecular Biology Reviews 64: 607-623.

Blader, I. J., I. D. Manger, and J. C. Boothroyd. 2001. Microarray analysis reveals previously unknown changes in Toxoplasma gondiiinfected human cells. Journal of Biological Chemistry 276: 24223 24231

, AND J. P. SAEIJ. 2009. Communication between Toxoplasma gondi and its host: Impact on parasite growth, development, immune evasion, and virulence. Acta Pathologica, Microbiologica et Immunologica 117: 458-476.

Blume-Jensen, P., R. Janknecht, And T. Hunter. 1998. The kit receptor promotes cell survival via activation of PI 3-kinase and subsequent Akt-mediated phosphorylation of Bad on Ser136. Current Biology 8: 779-782.

Bohne, W., J. Heesemann, And U. Gross. 1993. Induction of bradyzoitespecific Toxoplasma gondii antigens in $\gamma$ interferon-treated mouse macrophages. Infection and Immunity 61: 1141-1145.

Boothroyd, J. C., And J. F. Dubremetz. 2008. Kiss and spit: The dual roles of Toxoplasma rhoptries. Nature Reviews Microbiology 6: 79-88.

Breinich, M. S., D. J. Ferguson, B. J. Foth, G. G. van Dooren, M Lebrun, D. V. Quon, B. Striepen, P. J. Bradley, F. Frischknecht, V. B. Carruthers, and M. Meissner. 2009. A dynamin is required for the biogenesis of secretory organelles in Toxoplasma gondii. Current Biology 19: 277-286.

Brunet, J., A. W. Pfaff, A. Abidi, M. Unoki, Y. Nakamura, M. Guinard, J. P. Klein, E. Candolfi, and M. Mousli. 2008 Toxoplasma gondii exploits UHRF1 and induces host cell cycle arrest at G2 to enable its proliferation. Cellular Microbiology 10: 908-920.

Buitrago-Rey, R., J. Olarte, and J. E. Gomez-Marin. 2002. Evaluation of two inhibitors of invasion: LY311727 [3-(3-acetamide-1-benzyl-2ethyl-indolyl-5-oxy) propane phosphoric acid] and AEBSF [4-(2aminoethyl)-benzenesulphonyl fluoride] in acute murine toxoplasmosis. Journal of Antimicrobial Chemotherapy 49: 871-874.

Caldas, L. A., W. de Souza, And M. Attias. 2007. Calcium ionophoreinduced egress of Toxoplasma gondii shortly after host cell invasion. Veterinary Parasitology 147: 210-220.

Cao, J., O. Kaneko, A. Thongkukiatkul, M. Tachibana, H. Otsuki, Q GAO, T. TsuboI, And M. ToRII. 2009. Rhoptry neck protein RON2 forms a complex with microneme protein AMA1 in Plasmodium falciparum merozoites. International Journal for Parasitology 58: 29-35.

Chandramohanadas, R., P. H. Davis, D. P. Beiting, M. B. Harbut, C. Darling, G. Velmourougane, M. Y. Lee, P. A. Greer, D. S. Roos, and D. C. Greenbaum. 2009. Apicomplexan parasites co-opt host calpains to facilitate their escape from infected cells. Science 324: 794 797.

Charron, A. J., And L. D. Sibley. 2002. Host cells: Mobilizable lipid resources for the intracellular parasite Toxoplasma gondii. Journal of Cell Science 115: 3049-3059.

, AND - 2004. Molecular partitioning during host cell penetration by Toxoplasma gondii. Traffic 11: $855-867$.

Coppens, I., J. D. Dunn, J. D. Romano, M. Pypaert, H. Zhang, J. C. Boothroyd, AND K. A. JoIner. 2006. Toxoplasma gondii sequesters lysosomes from mammalian hosts in the vacuolar space. Cell 125: 261-274.

- A. P. Sinai, And K. A. Joiner. 2000. Toxoplasma gondii exploits host low-density lipoprotein receptor-mediated endocytosis for cholesterol acquisition. Journal of Cell Biology 149: 167-180. 
Crawford, M. J., N. Thomsen-Zieger, M. Ray, J. Schachtner, D. S. Roos, And F. SEeber. 2006. Toxoplasma gondii scavenges hostderived lipoic acid despite its de novo synthesis in the apicoplast. EMBO Journal 25: 3214-3222.

da Silva, C. V., E. A. da Silva, M. C. Cruz, P. C. Havrier, and R. A. Mortara. 2009. ARF6, PI3-kinase and host cell actin cytoskeleton in Toxoplasma gondii cell invasion. Biochemical and Biophysical Research Communications 378: 656-661.

Dobrowolski, J. M., And L. D. Sibley. 1996. Toxoplasma invasion of mammalian cells is powered by the actin cytoskeleton of the parasite. Cell 84: 933-939.

Dubey, J. P., D. S. Lindsay, And C. A. Speer. 1998. Structures of Toxoplasma gondii tachyzoites, bradyzoites, and sporozoites and biology and development of tissue cysts. Clinical Microbiology Reviews 11: 267-299.

Dubremetz, J. F., And J. D. Schwartzman. 1993. Subcellular organelles of Toxoplasma gondii and host cell invasion. Research in Immunology 144: 31-33.

Ferreira, S., T. M. De Carvalho, and W. De Souza. 2003. Protein phosphorylation during the process of interaction of Toxoplasma gondii with host cells. Journal of Submicroscopic Cytology and Pathology 35: 245-252.

Fox, B. A., J. P. Gigley, And D. J. BziK. 2004. Toxoplasma gondii lacks the enzymes required for de novo arginine biosynthesis and arginine starvation triggers cyst formation. International Journal for Parasitology 34: 323-331.

GaIl, M., U. Gross, And W. Bohne. 2001. Transcriptional profile of Toxoplasma gondii-infected human fibroblasts as revealed by genearray hybridization. Molecular Genetics and Genomics 265: 905-912.

Goebel, S., U. Gross, and C. G. Luder. 2001. Inhibition of host cell apoptosis by Toxoplasma gondii is accompanied by reduced activation of the caspase cascade and alterations of poly (ADP-ribose) polymerase expression. Journal of Cell Science 114: 3495-3505.

Grimwood, J., J. R. Mineo, And L. H. Kasper. 1996. Attachment of Toxoplasma gondii to host cells is host cell cycle dependent. Infection and Immunity 64: 4099-4104.

Guimarães, E. V., L. de Carvalho, and H. S. Barbosa. 2008. Primary culture of skeletal muscle cells as a model for studies of Toxoplasma gondii cystogenesis. Journal of Parasitology 94: 72-83.

Hakansson, S., A. J. Charron, And L. D. Sibley. 2001. Toxoplasma vacuoles: A two step process of secretion and fusion forms the parasitophorous vacuole. EMBO Journal 20: 3132-3144.

Hippe, D., O. Lytovchenko, I. Schmitz, and C. G. Lüder. 2008. Fas/ CD95-mediated apoptosis of type II cells is blocked by Toxoplasma gondii primarily via interference with the mitochondrial amplification loop. Infection and Immunity 76: 2905-2912.

JoIner, K. A., And D. S. Roos. 2002. Secretory traffic in the eukaryotic parasite Toxoplasma gondii: Less is more. Journal of Cell Biology 157: $557-563$.

Kafsack, B. F., J. D. Pena, I. Coppens, S. Ravindran, J. C. Boothroyd, AND V. B. Carruthers. 2009. Rapid membrane disruption by a perforin-like protein facilitates parasite exit from host cells. Science 323: 530-533.

Kim, L., And E. Y. Denkers. 2006. Toxoplasma gondii triggers Gidependent PI 3-kinase signaling required for inhibition of host cell apoptosis. Journal of Cell Science 119: 2119-2126.

Kim, S. K., A. E. Fouts, And J. C. Boothroyd. 2007. Toxoplasma gondii dysregulates IFN-gamma-inducible gene expression in human fibroblasts: Insights from a genome-wide transcriptional profiling. Journal of Immunology 178: 5154-5165.

Kirkman, L. A., L. M.Weiss, and K. KIm. 2001. Cyclic nucleotide signaling in Toxoplasma gondii bradyzoite differentiation. Infection and Immunity 69: 148-153.

Laliberté, J., And V. B. Carruthers. 2008. Host cell manipulation by the human pathogen Toxoplasma gondii. Cellular and Molecular Life Sciences 65: 1900-1915.

Lang, C., M. Algner, N. Beinert, U. Gross, and C. G. Luder. 2006. Diverse mechanisms employed by Toxoplasma gondii to inhibit IFNgamma-induced major histocompatibility complex class II gene expression. Microbes and Infection 8: 1994-2005.

Lavine, M. D., and G. Arrizabalaga. 2009. Induction of mitotic S-phase of host and neighboring cells by Toxoplasma gondii enhances parasite invasion. Molecular and Biochemical Parasitology 164: 95-99.
LI, L., LI, X, AND J. YAN. 2008. Alterations of concentrations of calcium and arachidonic acid and agglutinations of microfilaments in host cells during Toxoplasma gondii invasion. Veterinary Parasitology 157: 21-33.

Lindsay, D. S., J. P. Dubey, B. L. Blagburn, and M. A. ToivioKinnuCAN. 1991. Examination of tissue cyst formation by Toxoplasma gondii in cell cultures using bradyzoites, tachyzoites, and sporozoites. Journal of Parasitology 77: 126-132.

, R. R. Mitschler, M. A. Toivio-Kinnucan, S. J. Upton, J. P. Dubey, And B. L. Blagburn. 1993. Association of host cell mitochondria with developing Toxoplasma gondii tissue cysts. American Journal of Veterinary Research 54: 1663-1667.

, M. A. Tolvio-Kinnucan, And B. L. Blagburn. 1993. Ultrastructural determination of cystogenesis by various Toxoplasma gondii isolates in cell culture. Journal of Parasitology 79: 289-292.

-, AND — 1998. Decoquinate induces tissue cyst formation by the RH strain of Toxoplasma gondii. Veterinary Parasitology 77: 75-81.

Lingelbach, K., And K. A. JoIner. 1998. The parasitophorous vacuole membrane surrounding Plasmodium and Toxoplasma: An unusual compartment in infected cells. Journal of Cell Science 111: 1467-1475

Lovett, J. L., N. Marchesini, S. N. Moreno, and L. D. Sibley. 2002. Toxoplasma gondii microneme secretion involves intracellular $\mathrm{Ca}(2+)$ release from inositol 1,4,5-triphosphate (IP(3))/ryanodine-sensitive stores. Journal of Biological Chemistry 277: 25870-25876.

, AND L. D. Sibley. 2003. Intracellular calcium stores in Toxoplasma gondii govern invasion of host cells. Journal of Cell Science 116: 3009-3016.

Luder, C. G., And U. Gross. 2005. Apoptosis and its modulation during infection with Toxoplasma gondii: Molecular mechanisms and role in pathogenesis. Current Topics in Microbiology and Immunology 289: 219-238.

Lyons, R. E., R. McLeod, And C. W. Roberts. 2002. Toxoplasma gondii tachyzoite-bradyzoite interconversion. Trends in Parasitology 18: 198-201.

Maclaren, A., M. Attias, and W. de Souza. 2004. Aspects of the early moments of interaction between tachyzoites of Toxoplasma gondii with neutrophils. Veterinary Parasitology 125: 301-312.

Melo, E. J., T. M. Carvalho, and W. De Souza. 2001. Behaviour of microtubules in cells infected with Toxoplasma gondii. Biocell 25: 53-59.

Melzer, T., A. Duffy, L. M. Weiss, and S. K. Halonen. 2008. The Gamma interferon (IFN- $\gamma$ )-inducible GTP-binding protein IGTP is necessary for Toxoplasma vacuolar disruption and induces parasite egression in IFN- $\gamma$-stimulated astrocytes. Infection and Immunity 76: 4883-4894.

Molestina, R. E., N. El-Guendy, And A. P. Sinai. 2008. Infection with Toxoplasma gondii results in dysregulation of the host cell cycle. Cellular Microbiology 10: 1153-1165.

, T. M. Payne, I. Coppens, And A. P. Sinai. 2003. Activation of NF-kappaB by Toxoplasma gondii correlates with increased expression of antiapoptotic genes and localization of phosphorylated IkappaB to the parasitophorous vacuole membrane. Journal of Cell Science 116: 4359-4371.

Mordue, D. G., N. Desai, And M. Dustin. 1999. Invasion by Toxoplasma gondii establishes a moving junction that selectively excludes host cell plasma membrane proteins on the basis of their membrane anchoring. Journal of Experimental Medicine 190: 1783-1792.

- S. Hakansson, I. Niesman, And L. D. Sibley. 1999. Toxoplasma gondii resides in a vacuole that avoids fusion with host cell endocytic and exocytic vesicular trafficking pathways. Experimental Parasitology 92: 87-99.

Narasimhan, J., B. R. Joyce, A. Naguleswaran, A. T. Smith, M. R. Livingston, S. E. Dixon, I. Coppens, R. C. Wek, and W. J. Sullivan. 2008. Translation regulation by eukaryotic initiation factor-2 kinases in the development of latent cysts in Toxoplasma gondii. Journal of Biological Chemistry 283: 16591-16601.

Nash, P. B., M. B. Purner, R.P. Leon, P. Clarke, R. C. Duke, and T. J. CurIEl. 1998. Toxoplasma gondii infected cells are resistant to multiple inducers of apoptosis. Journal of Immunology 160: 1824 1830.

Pacheco-Soares, C., And W. De SouzA. 2000. Labeled probes inserted in the macrophage membrane are transferred to the parasite surface and 
internalized during cell invasion by Toxoplasma gondii. Parasitology Research 86: 11-17.

Patra, A. K., S. Y. NA, and U. Bommhardt. 2004. Active protein kinase $\mathrm{B}$ regulates TCR responsiveness by modulating cytoplasmic-nuclear localization of NFAT and NF-kappa B proteins. Journal of Immunology 172: 4812-4820.

Payne, T. M., R. E. Molestina, And A. P. Sinai. 2003. Inhibition of caspase activation and a requirement for $\mathrm{NF}-\mathrm{\kappa B}$ function in the Toxoplasma gondii-mediated blockade of host apoptosis. Journal of Cell Science 116: 4345-4358.

Persson, E. K., A. M.Agnarson, H. Lambert, N. Hitziger, H. Yagita, B. J. Chambers, A. Barragan, and A. Grandien. 2007.Death receptor ligation or exposure to perforin trigger rapid egress of the intracellular parasite Toxoplasma gondii. Journal of Immunology 179: $8357-8365$.

Pingret, L., J. M. Millot, S. Sharonov, A. Bonhomme, M. Manfait, AND J. M. PINON.1996. Relationship between intracellular free calcium concentrations and the intracellular development of Toxoplasma gondii. Journal of Histochemistry and Cytochemistry 44: $1123-1129$.

Radke, J. R., M. N. Guerini, M. Jerome, and M. W. White. 2003. A change in the premitotic period of the cell cycle is associated with bradyzoite differentiation in Toxoplasma gondii. Molecular and Biochemical Parasitology 131: 119-127.

Rodriguez, A., M. G. Rioult, A. Ora, and N. W. Andrews. 1995. A trypanosome-soluble factor induces IP3 formation, intracellular Ca2+ mobilization and microfilament rearrangement in host cells. Journal of Cell Biology 129: 1263-1273.

Ronco, A. M., P. F. Moraga, and M. N. Llanos. 2002. Arachidonic acid release from rat Leydig cells: The involvement of $\mathrm{G}$ protein, phospholipase A2 and regulation of cAMP production. Journal of Endocrinology 172: 95-104.

Saffer, L. D., O. Mercereau-Puijalon, J. F. Dubremetz, and J. D. SChWARTZMAn. 1992. Localization of a Toxoplasma gondii rhoptry protein by immunoelectron microscopy during and after host cell penetration. Journal of Protozoology 39: 526-530.

SAlter, M. W., AND J. L. Hicks. 1995. ATP causes release of intracellular $\mathrm{Ca} 2+$ via the phospholipase $\mathrm{C}$ beta/IP3 pathway in astrocytes from the dorsal spinal cord. Journal of Neuroscience 15: 2961-2971.

Scheid, M. P., AND J. R. Woodget. 2001. PKB/Akt: Functional insights from genetic models. Nature Reviews Molecular Cell Biology 2: 760 768.

Schwab, J. C., C. J. M. Beckers, and K. A. Joiner. 1994. The parasitophorous vacuole membrane surrounding intracellular Toxoplasma gondii functions as a molecular sieve. Proceedings of the National Academy of Sciences USA 91: 509-513.
Sehgal, A., S. Bettiol, M. Pypaert, M. R. Wenk, A. Kaasch, I. J. Blader, K. A. Joiner, And I. Coppens. 2005. Peculiarities of host cholesterol transport to the unique intracellular vacuole containing Toxoplasma. Traffic 6:1 125-1141.

Sibley, L. D., J. M. Dobrowolski, J. H. Morisaki, and J. E. Heuser. 1994. Invasion and intracellular survival by Toxoplasma gondii. In Strategies for intracellular survival of microbes, D. G. Russell (ed.). Bailliere Tindall, London, U.K., p. 245-264.

, I. R. Niesman, S. F. Parmley, and M. F. Cesbron-Delauw. 1995. Regulated secretion of multi-lamellar vesicles leads to formation of a tubule-vesicular network in host-cell vacuoles occupied by Toxoplasma gondii. Journal of Cell Science 108: 1669-1677.

SinaI, A. P., AND K. A. JoIner. 2001. The Toxoplasma gondii protein ROP2 mediates host organelle association with the parasitophorous vacuole membrane. Journal of Cell Biology 154: 95-108.

- P. Webster, And K. A. Joiner. 1997. Association of host cell endoplasmic reticulum and mitochondria with the Toxoplasma gondii parasitophorous vacuole membrane: A high affinity interaction. Journal of Cell Science 110: 2117-2128.

Soete, M., D. Camus, and J. F. Dubremetz. 1994. Experimental induction of bradyzoite-specific antigen expression and cyst formation by the RH strain of Toxoplasma gondii in vitro. Experimental Parasitology 78: $361-370$.

Straub, K., S. Cheng, C. Sohn, and P. Bradley. 2009. Novel components of the apicomplexan moving junction reveal conserved and coccidianrestricted elements. Cellular Microbiology 11: 590-603.

Suzuki, Y., F. K. Conley, and J. S. Remington. 1989. Importance of endogenous IFN- $\gamma$ for prevention of toxoplasmic encephalitis in mice. Journal of Immunology 143: 2045-2050.

Sweeney, K. R., N. S. Morrissette, S. Lachapelle, and I. J. Blader. 2010. Host cell invasion by Toxoplasma gondii is temporally regulated by the host microtubule cytoskeleton. Eukaryotic Cell 9: 1680-1689.

Vutova, P., M. Wirth, D. Hippe. U. Gross, K. Schulze-Osthoff, I. SChMitz, AND C. G. Lüder. 2007. Toxoplasma gondii inhibits Fas/ CD95-triggered cell death by inducing aberrant processing and degradation of caspase 8. Cellular Microbiology 9: 1556-1570.

Walker, M. E., E. E. HJort, S. S. Smitha, A. Tripathi, J. E. Hornick, E. H. Hinchcliffe, W. Archer, and K. M. Hager. 2008. Toxoplasma gondii actively remodels the microtubule network in host cells. Microbes and Infection 10: 1440-1449.

Yang, Z. Z., O. Tschopp, A. Baudry, B. Dümmler, D. Hynx, and B. A Hemmings. 2004. Physiological functions of protein kinase B/Akt. Biochemical Society Transactions 32: 350-354.

Zimmermann, S., P. J. Murray, K. Heeg, and A. H. Dalpke. 2006. Induction of suppressor of cytokine signaling-1 by Toxoplasma gondii contributes to immune evasion in macrophages by blocking IFNgamma signaling. Journal of Immunology 176: 1840-1847. 